\title{
EDITORIAL
}

\section{COVID -19 INFECTION, THE PANDEMIC OF THE CENTURY-ARE WE PREPARED YET?}

\author{
Md Robed Amin', Quazi Tarikul Islam²
}

From the desk of editorial office

In late December 2019, a cluster of patients with pneumonia of unknown cause was linked to a seafood wholesale market in Wuhan, the capital of Hubei province and is the seventh-largest city in China, with a population $>11$ million. A case control study revealed a novel virus linked with these pneumonia cases. A previously unknown beta corona virus was discovered initially named $2019-\mathrm{nCoV}$ on $12^{\text {th }}$ January ${ }^{1}$. WHO lately revised the virus name as SARS CoV-2 and named the disease as COVID-19. Although different from both MERS-CoV and SARS-CoV in characteristics, SARS CoV-2 is the seventh member of the family of coronaviruses that infect humans have phylogenetic similarities with SARS CoV and hence the name was changed ${ }^{1}$.

COVID-19 is a zoonotic virus. From phylogenetics analyses undertaken with available full genome sequences, bats appear to be the reservoir of COVID19 virus $^{2.3}$ while the intermediate host was suggested as pangolin. COVID-19 is transmitted via droplets and fomites during close unprotected contact between an infector and infectee $e^{4,5}$. Airborne spread has been reported only in context of aerosol generating procedure and very close gatherings with recent report for COVID$19^{4,5}$ but it is not believed to be a major driver of transmission based on available evidence. There has been shedding of virus in stool of infected person reported in few case reports. Human-to-human transmission of the COVID-19 virus is largely occurring in families in china and also observed in all over Europe especially Italy, United kingdom, France, Belgium, iran ${ }^{1,3}$ and now in USA with huge transmission with mortality and morbidity. The containment strategy of lockdown leads to such transmission, but it also halts the widely community transmission in a country. Preliminary studies ongoing in Guangdong estimate the secondary attack rate in households ranges from $3-10 \%{ }^{5}$. China has a policy of meticulous case and contact identification for COVID-19 and are tracing tens of thousands of contacts a day. Contact follow up is painstaking, with a high percentage of identified close contacts completing medical observation. Between $1 \%$ and $5 \%$ of contacts were subsequently laboratory confirmed cases of COVID-19, depending on location ${ }^{5}$.

There has been mutation in SARS CoV-2 virus as early as in January in Wuhan city. The early form of $\mathrm{S}$ converts into L form which were associated with extensive community transmission and severity and death. The huge mortality was due to ARDS and the involvement of cardiac arrest as cardiac myocyte ACER2 showed sensitivity to L form. The extensive mitigation effort by China then halts the L form transmission dynamics and both $\mathrm{S}$ form and $\mathrm{L}$ form are circulating outside of china now. It seems Italy and Iran both have more $\mathrm{L}$ form than $\mathrm{S}$ form of mutated virus as the transmission and fatality suggest the experience of Wuhan. It is not sure whether any other mutation happens to virus or not at this moment. The mutation can pass through both way of devastation or calmness while the reassortment with other viral gene is also a high possibility of more evolving situation of SARS-coV 2. Currently many other mutation has been identified.

Testing for COVID-19 from routine respiratory disease surveillance systems to explore if COVID-19 is circulating more broadly and undetected in the community is important for any country including Bangladesh. These systems could include RT-PCR testing of COVID-19 virus in influenza-like-illness (ILI) and severe acute respiratory infection (SARI) surveillance systems, as well as testing of results among all visitors to fever clinics. As Bangladesh already declared two hundred thousands of cases and worldwide pandemic is extensively observed from community to community, its prudent for us to explore the CoVID-19 case detection through RT-PCR and suology while continuing regular ILI and SARI surveillance. As COVID-19 is a newly identified pathogen, there is no known pre-existing immunity in humans. Everyone is assumed to be susceptible, although increasing susceptibility to infection depends on risk factors like Diabetes, hypertension, immune suppressed states etc.

That is why aggressive case and contact identification, isolation and management and extreme social distancing, should be implemented to interrupt the

Bangladesh J Medicine 2020; 31 : 43-45

DOI: https://doi.org/10.3329/bjm.v31i2.48530 
chains of transmission nationwide. Of note, the highly clustered nature of local transmission may explain a relatively high RO (2.5) in the absence of interventions in China and low confirmed case counts with intense quarantine and social distancing measures. The pandemic pyramids explains the scenario perfectly in China indicating the numbers of cases were actually far more than confirmed while the severe cases were all counted as they took service from designed hospital.

Among the Health care worker infections, most were identified early in the outbreak in Wuhan when supplies and experience with the new disease was lower. But later discrete and limited instances of nosocomial outbreak within health care settings and amongst health care workers appear due to extensive availability of Personal protective equipment(PPE). But the scenario of such ideal standard may not be observed in Bangladesh as HCW here are not acquainted with PPE and there is gross lack of availability and quality of such equipment in case of huge public health transmission. The close proximity and contact among people in closed living settings and the potential for environmental contamination are important factors, which could amplify transmission in CoVID 19 infection which all are alarming for Bangladesh. Data on individuals aged 18 years old and under suggest that there is a relatively low attack rate in this age group $(2.4 \%$ of all reported cases in China). it is not possible yet to determine the extent of infection among children, what role children play in transmission, whether children are less susceptible or if they present differently clinically.

Typical signs and symptoms include: fever (87.9\%), dry cough $(67.7 \%)$, fatigue $(38.1 \%)$, sputum production $(33.4 \%)$, shortness of breath $(18.6 \%)$, sore throat $(13.9 \%)$, headache $(13.6 \%)$, myalgia or arthralgia $(14.8 \%)$, chills $(11.4 \%)$, nausea or vomiting $(5.0 \%)$, nasal congestion (4.8\%), diarrhea (3.7\%), and hemoptysis $(0.9 \%)$, and conjunctival congestion $(0.8 \%)^{6}$. People with COVID-19 generally develop signs and symptoms, including mild respiratory symptoms and fever, on an average of 5-6 days after infection (mean incubation period 5-6 days, range 1-14 days). About $80 \%$ cases are having mild illness, $15 \%$ cases were severe and hence need hospitalization and 5\% case need ICU support due to development of $\operatorname{ARDS}^{6,7,8}$. The crude death rate in china was $2.4 \%$ and more the ages, more was the fatality. The big problem is around $20 \%$ cases need hospital care with many ICU support. ${ }^{8}$ If the transmission is heavy, then this seemingly less danger disease can give serious toll to the health system and infrastructure of a country. That has been observed in Italy and Iran and also in many developed country in Europe and America who doesn't think beforehand. The same problem will be is an alarm for Bangladesh as our health facility will not cover these intense burdens of hospitalization and ICU care. The prejudice of deadly for old concept is also not always correct as Italy \& USA is observing recently severity and fatality in young groups of people. Asymptomatic infection has been reported, but the majority of the relatively rare cases who are asymptomatic on the date of identification/report went on to develop disease. The proportion of truly asymptomatic infections is unclear but appears to be relatively not common and does not appear to be a major driver of transmission. Those with underlying conditions such as older age, hypertension, diabetes, cardiovascular disease, chronic respiratory disease, immunosuppressive agent taker and cancer are the major risk factor that has been observed globally ${ }^{8}$.

Bangladesh is now facing a new viral disease which is nonimmune to all of its citizen and with unpredictable course and future. Up to the date (30.6.2020), Bangladesh is having around 150000 patients with 2000 death and trends is increasing ${ }^{9}$. Bangladesh is in the middle of lockdown now for a month and hospitals are just about to be prepared and facing extra burden due to COVID-19 cases. The lockdown is not perfectly observed, and we had few big upset like garments worker walking for unplanned BGMEA decision, a poor religious burial activity of a leader with thousands of people and again a surge of unplanned opening of offices and industries. The hospital preparedness is completely in disarray with inappropriate infrastructure, poor planning and lack of personal protective equipment, safety issue and lack of coordination with health care worker and administration. We are also far behind of test which has been repeatedly suggested by WHO with poor capacity development and approach and no system at all for capturing pre symptomatic or asymptomatic sates. The primary, secondary and tertiary health care system (public and private) all are in confused states due to lack of uniform command system for pandemic. The contact tracing is now in jeopardy and makes transmission in upload mood and proper isolation (for case) or quarantine (for contact) in home and institutional are not appropriately in placed. The country is in peak of level 4 but the administration, surveillance, case management, research, contact management, mitigation and containment process, community engagement all are in premature stage. Until we cannot engage the community, it will be simply impossible to mitigate the COVID-19 from Bangladesh. We need planning to understand the preparedness for pandemic rather self-belief of that we are prepared. 


\section{References:}

1. WHO. Novel coronavirus - Jan 17, 2020. http:// www.who.int/csr/don/17-january-2020-novelcoronavirusjapan-ex-china/en/ (accessed April 19, 2020).

2. Roujian Lu, Xiang Zhao, Juan Li, PeihuaNiu, Bo Yang, Honglong Wu et al. Genomic characterisation and epidemiology of 2019 novel coronavirus: implications for virus origins and receptor binding. the lancet.com, January 29, 2020 https://doi.org/10.1016/ S01406736(20)30251-8

3. Ksiazek TG, Erdman D, Goldsmith CS, et al. A novel coronavirus associated with severe acute respiratory syndrome. N Engl J Med 2003; 348: 1953-66. https:// doi.org/10.1056/NEJMoa030781 PMid:12690092

4. Kuiken T, Fouchier RAM, Schutten M, et al. Newly discovered coronavirus as the primary cause of severe acute respiratory syndrome. Lancet 2003; 362: 26370. https://doi.org/10.1016/S0140-6736(03)13967-0
5. DC. First travel-related case of 2019 novel coronavirus detected in United States. Jan 21, 2020. https:// www.cdc.gov/media/ releases/2020/p0121-novelcoronavirus-travel-case.html (accessed Jan 23, 2020).

6. Tan W, Zhao X, Ma X, et al. A novel coronavirus genome identified in a cluster of pneumonia cases - Wuhan, China 2019"2020. http://weekly.chinacdc.cn/en/ article/id/a3907201-f64f-4154-a19e4253b453d10c (accessed Jan 23, 2020).

7. Chaolin Huang, Yeming Wang, Xingwang Li, Lili Ren, Jianping Zhao, Yi Hu et al.Clinical features of patients infected with 2019 novel coronavirus in Wuhan, China,www.thelancet.com Published online January 24, 2020 https://doi.org/10.1016/S01406736(20)30183-5. https://doi.org/10.1016/S01406736(20)30183-5

8. Report of the WHO-China Joint Mission on Coronavirus Disease 2019 (COVID-19), 16-24 february,2020

9. Report of corona update cell, IEDCR, DGHS, Bangladesh, dated-late june, 2020, www.iedcr,gov.bd 